\title{
Strategies for writing a successful National Institutes of Health grant proposal for the early-career neurointerventionalist
}

\author{
Peter Kan (D) , ${ }^{1}$ Maxim Mokin (D) ,' ${ }^{2}$ William J Mack, ${ }^{3}$ Robert M Starke, ${ }^{4,5}$ \\ Kevin N Sheth, ${ }^{6}$ Felipe C Albuquerque, ${ }^{7}$ Michael R Levitt (i) ${ }^{8}$
}

${ }^{1}$ Neurosurgery, The University of Texas Medical Branch at Galveston, Galveston, Texas, USA

${ }^{2}$ Neurosurgery, University of South Florida, Tampa, Florida, USA

${ }^{3}$ Neurosurgery, University of Southern California, Los Angeles, California, USA ${ }^{4}$ Neurological Surgery, University of Miami Miller School of Medicine, Miami Beach, Florida, USA

${ }^{5}$ University of Miami Miller School of Medicine, Miami Florida, USA

${ }^{6}$ Department of Neurology, Yale University, New Haven, Connecticut, USA

${ }^{7}$ Department of Neurosurgery, Barrow Neurological Institute, Phoenix, Arizona, USA ${ }^{8}$ Neurological Surgery, University of Washington School of Medicine, Seattle, Washington, USA

\section{Correspondence to}

Dr Peter Kan, Neurosurgery, The University of Texas Medical Branch at Galveston, Galveston, Texas, USA; ptkan@utmb.edu

Accepted 20 October 2020 Published Online First 25 November 2020

\section{Linked}

- http://dx.doi.org/10.1136/ neurintsurg-2020-016743

Check for updates

(c) Author(s) (or their employer(s)) 2021. No commercial re-use. See rights and permissions. Published by BMJ.

To cite: Kan $\mathrm{P}$, Mokin M

Mack WJ, et al.

J Neurolntervent Surg

2021:13:283-286

\section{ABSTRACT}

Objective The goal of this article is to provide a succinct review of the key components of a NIH grant application and the $\mathrm{NIH}$ reviewprocess for the early career neurointerventionalist.

Methods The authors reviewed NIH rules and regulations and also reflected on their own collective experiencein writing $\mathrm{NIH}$ grant proposals in the area of cerebrovascular disease andneurointerventional surgery. Results Key components of theresearch strategy include specific aims, significance, innovation and approach. The specific aims page is the most important page of the application and should be written first. The NIH review isbased on these key components along with an assessment of the appropriatenessof the investigators and environment for the research.

Conclusion Detailed knowledge ofthe key components of the research grant is critical to a successful application. The information in the article may aid in the grant writing for early careerneurointerventionalists.

\section{INTRODUCTION}

The outline and structure of a grant proposal varies substantially depending on the type of grant and its funding agency. The National Institutes of Health (NIH) grant application often represents the most comprehensive application structure, which will be the primary subject of this review. Here we outline the key parts of the NIH grant, along with critical components of each.

For the most commonly-sought research grant (R01), ${ }^{1}$ the Specific Aims is a maximum of 1 page and the Research Strategy (Significance, Innovation, and Approach) cannot exceed 12 pages in total. Given the page limit for the latter, we find the use of tables and figures to be extremely helpful. In general, we recommend an average of one figure or table per page to help summarize the data or experiments.

\section{Specific aims}

This is the most important page of the research grant and should be written early, with constant refinement. It summarizes the significance, innovation, impact, approach, and investigators of the entire research proposal, all within a single page, which provides the initial and sometimes the only impression of the overall project to the reviewer. It is often used by the NIH Program Officer (PO) to assign a proposal to a study section. Critical components include a well-defined problem of significance, a gap in knowledge, an innovative hypothesis with a strong scientific premise, well-structured aims to test the hypothesis, and a summary of overall impact. Each grant usually consists of 2-3 aims and the aims must be supportive but independent. A well-written Specific Aims page is clear, straightforward, demonstrates the importance of the problem as well as the method of solving it, and provides a set of deliverables or success criteria on a realistic timeline. ${ }^{2}$

\section{Significance}

The Significance section should highlight the scientific problem important to the NIH mission or a critical barrier in the field and is similar to the introduction of a scientific paper. The importance of the problem is often introduced in 'scope of the problem' where the incidence, morbidity/mortality, and financial burden of the health problem are introduced. This section must also present a strong foundation for the project based on rigorous prior scientific research from the literature or the researcher's own preliminary data (termed the 'scientific premise'). Finally, measurable improvement in scientific knowledge, technical capability, and clinical practice as a result of the proposal should be described here as deliverables (impact). In general, the Significance section requires $3-5$ pages in a 12 -page proposal. ${ }^{3}$

\section{Innovation}

This is a short section that should only take about half a page. Three critical elements are conceptual innovation (how does the application challenge the current paradigm), technical innovation (new techniques and/or instruments), and the applicability of these innovations (to one field only or more broadly to multiple disciplines). Examples of highly innovative proposals include the application of newly developed technology to an existing problem, approaching an existing problem from a novel perspective, or applying a technique from a disparate scientific discipline to address a clinical question. An incremental step forward with existing technology and well-described mechanisms, especially those that have already been investigated and previously published, is not considered innovative. ${ }^{4}$ 


\section{Approach}

This part of the Research Strategy details the experiments, akin to the methods section of a scientific paper. The approach must (1) include rigorous methodologies to achieve unbiased results; (2) provide explanation of how biological variables (eg, sex and ethnicity) are factored into research design and analyses; (3) consider all necessary controls; and (4) discuss how data will be collected, analyzed, and interpreted. ${ }^{5}$

The approach is usually divided according to aims, each of which should be a self-contained experiment or set of experiments to address a fundamental component of the overall scientific problem that the grant is attempting to solve. Each aim includes the following subsections: (1) introduction/rationale (here is what we want to do and why); (2) preliminary data (they reinforce the scientific premise of the aim, support the hypothesis, and establish feasibility; and (3) research design with sound methodologies. Statistical analysis should include power analysis and consideration of experiments or stratification based on gender and race. Power analysis/sample size calculations should take into account the study effect, tolerated error, interim analysis, and loss to follow-up; (4) expected results (to ensure a good understanding of the experiments); and (5) a brief explanation of potential pitfalls/alternative strategies (to de-risk the proposed aim). Often, each aim will include its own hypothesis to be tested; if an experiment or a set of tests is required but does not completely address a hypothesis, then those experiments or tests are considered 'tasks' or 'sub-aims'.

\section{OTHER IMPORTANT DOCUMENTS IN THE NIH APPLICATION Project summary/abstract}

A concise summary (maximum 30 lines) of the proposed work written for other researchers in the same or a related field. It usually includes specific aims, research design, and methodology of the proposal. ${ }^{6}$

\section{Project narrative}

This consists of 2-3 (maximum) sentences written for a layperson and will be made publicly available on funding. It highlights to the public how the project aligns with the NIH mission. ${ }^{6}$

\section{Biosketch}

The biosketch contains a personal statement with up to four publications, positions, and honors, contributions to science (up to five contributions with a maximum of four publications per contribution), link to full bibliography, research support, and current and completed grants/research projects. It should highlight the alignment of the career theme with the proposal. Personal statements should be tailored to the specific project the researcher is applying for and should emphasize researcher independence, expertise, up-to-date bibliographic information, and current research support. The biosketch requires a specific format that is updated every few years and should not be confused with a curriculum vitae (CV) that some grant agencies other than NIH may require instead. ${ }^{7}$

\section{Facilities, equipment, and other resources}

This document outlines the availability and capability of institutional resources to perform the work proposed. Details include experimental capacities, laboratory square footage, relative proximity between the different resources, and specifics relevant for reproducibility of the experiments. Photographs are often a good way to communicate the available resources. A well-thought-out and designed research project may receive a poor score if the applicant fails to demonstrate the feasibility of institutional support or infrastructure necessary to successful complete the goals and aims of the proposal. ${ }^{8}$

\section{Letters of support}

These are proofs of collaboration and endorsement. Every collaborator should provide a letter of support. The support should not be conditional on receiving an award. Letters should be solicited early in the research planning process, be submitted on official letterhead, and outline either (1) a commitment to perform a specific aspect or aspects of the research proposal or (2) general support of the research endeavor (such as by a departmental chair or institute director). ${ }^{9}$

\section{Vertebrate animals}

This section includes a concise description of the proposed animal procedures to be used and identifies species, strains, age, sex, weight, and total number of animals based on power analyses outlined in the Research Strategy. Justification that specific species are appropriate for the proposed research and why research goals cannot be accomplished using an alternate model are provided. Interventions to minimize pain and distress such as analgesia, anesthesia, sedation, and euthanasia are also described. ${ }^{10}$

\section{Human subjects (if applicable)}

$\mathrm{NIH}$ is committed to increased representation of women, racial/ ethnic minorities, persons with disabilities, and other individuals who have been traditionally underrepresented in science. The grant proposal must describe how individuals from these underrepresented groups will be included in all aspects of the study (eg, 'Inclusion of women and minorities' and 'Inclusion of children' sections) and mechanisms to increase their participation (eg, consent forms in different languages and an ethnically diverse research team). For clinical trials, the 'Human Subject Section' should also include protection of human subjects, recruitment and retention plan, study timeline, single institutional review board plan for multisite study (eg, Smart IRB), data safety monitoring plan, structure of the study team, statistical design and power, investigative device or investigative drug status from the Food and Drug Administration, clinical milestone plan (start-up activities, go/no-go enrollment milestones, analysis, and reporting), and results dissemination plan. Common concerns include inadequate details concerning source of data, excessive physical/psychological risks to study subjects, lack of data confidentiality, invalid informed consent process, and unexpected findings. ${ }^{11}$

\section{Resource sharing plan}

Publications and presentations of the results are common methods of data sharing and should be fully compliant with the voluntary NIH Public Access Policy. In general, a data sharing plan should outline what kinds of data will be shared, who will have access to them and when, where are the data stored, and how will researchers locate and access the data. After analyses are complete, a de-identified dataset suitable for analysis needs to be submitted to the NIH. ${ }^{12}$

\section{BUDGET AND JUSTIFICATIONS}

The budget should be linked to facilitate achievement of the specific aims. The general budget for a typical R01 is $\$ 250000$ per year of direct cost (DC) over 5 years, excluding indirect costs (additional amount paid to the institution by the NIH to 
cover cost of the institution). Budgets can be modular or nonmodular. ${ }^{13}$ A modular format requests $\$ 250000$ or less per year in DC whereas a non-modular format requests more than $\$ 250000$ per year in DC. We recommend a modular budget for the first R01 application of the early-career interventionalist to convey fiscal consideration and a less risky proposal. Should a project need more than $\$ 250000$ in DC per year, additional budget details and a strong budget justification are required. The main categories in a budget and justification include Personnel, Supplies, Tuition, Equipment, Travel, and Publications. The following paragraphs provide some guidance and estimates for the application.

\section{Personnel and salary}

In general, one aim is equivalent to one thesis and requires a full-time graduate student for 4-5 years or one full-time postdoctoral researcher for 2.5-3 years. The principal investigator (PI) generally has between $10 \%$ and $30 \%$ effort while co-investigators and senior personnel have effort of between $5 \%$ and $15 \%$. Note that the salary cap for the NIH in 2020 is $\$ 197300,{ }^{14}$ so the percentage of salary (based on effort) paid for by the NIH will not exceed this limit, regardless of whether the investigator is paid a higher salary at his/her institution. This is important for the neurointerventionalist because it does not cover salary in general (eg, 30\% effort for the PI is only $\$ 59$ 190). Clinical trials will also need to budget for a project manager, safety monitor, research assistants, and coordinators at the main coordinating sites for multicenter trials.

\section{Supplies and equipment}

Supplies (such as reagents, software, engineering materials, or chemicals) should be matched to the aims, and separate justifications are given for large equipment purchases, which are often more permanent and more expensive than supplies.

\section{Travel}

Keeping conference travel cost low is recommended. A travel budget includes number of travelers, destination, number of days, per diem rates, and airfare costs.

\section{Publications}

On average, 8-12 publications are required for a competitive R01 renewal, and the average cost of publication including public access charges is $\$ 3000$ (though some journals provide public access for free in cases of federally-funded research). A good estimation is to budget for at least one major publication for every $\$ 120000$ of direct cost.

\section{NIH REVIEW CRITERIA, SCORING, AND HOW TO ALIGN YOUR APPLICATION}

The NIH review criteria follow the format of the Research Strategy. ${ }^{15}$ They include (1) significance of the research. It takes into account the importance of the problem studied, the critical barrier in the field that the study is trying to overcome, and the advancement of scientific knowledge if the aims were achieved; (2) innovation looking for novel concepts or approaches that challenge existing paradigms; (3) approach with sound design, methods, and analyses appropriate to the aims of the project along with acknowledgement of potential problems areas and consideration of alternatives; (4) investigators with appropriate level of training, experience, and complementary expertise; and (5) environment with adequate institutional support and laboratory equipment.
After submission, each application is assigned to a study section ${ }^{16}$ that consists of about 20 people from a variety of clinical, research, and industry backgrounds, and overseen by a Scientific Review Officer (from the NIH) and a Study Section Chair (an experienced reviewer). Each grant is initially assigned to an in-depth review by three reviewers (and occasionally more depending on the grant mechanism). Prior to the scheduled meeting date for the study section, each reviewer provides a thorough summary of the grant, including the criteria above, as well as an overall impact score, all on a scale of 1 (best) to 9 (worst). After all scores are submitted, a threshold of impact scores is determined. Applications scored above (worse than) the threshold are not discussed by the entire panel, but rather 'triaged' as not suitable for consideration of funding. If the grant is below (better than) the threshold, it is presented by the three in-depth reviewers to all study section members, after which all submit a final impact score. Scores are averaged and multiplied by 10 to derive an overall impact score, as well as a percentile rank compared with other submitted applications. Impact scores of 10 to 30 (corresponding to percentiles of $15 \%$ or less) are most likely to be funded and scores greater than 45 are rarely funded, though the payline below which applications are funded varies by grant mechanism, year, and by NIH institute.

Significance, innovation, and approach are mandatory sections of the Research Strategy. To align the rest of an application with the scoring criteria, attention should be paid to the other documents outlined above. For example, biosketches, personal statement, and letters of support are important to the assessment of the investigators and facilities, equipment, and other resources is important to grading the environment.

\section{OUTCOMES}

\section{Funded}

The general payline for the National Institute of Neurological Disorders and Stroke (NINDS) in 2020 is 16\% for all investigators and $25 \%$ for early stage investigators (ESIs). ${ }^{1}$

\section{Queuing}

This occurs when the impact score is just outside of payline.

\section{Discussed and scored}

The impact score is below the threshold. The significance and innovation scores are usually much lower (better) than the approach scores in these applications.

\section{Not discussed}

These are applications with impact scores above the threshold. The review comments include reasons why scores were high (poor), which can provide a roadmap for revisions to improve a grant application's chances on resubmission. It is important to be persistent as an application that was not discussed at one session can be funded at the next if appropriate revisions are made.

\section{COMMON REASONS NOT TO GET FUNDED}

Many applications are unfunded due to being too ambitious with a wide scope, leading to reviewers' concern about feasibility of completion of the work in the allotted timeframe. Other applications fail because aims are considered 'dependent', meaning that the success of one aim is predicated on the success of another aim; if a single aim fails, then the entire proposal will fail. This is considered too risky by NIH criteria, and should be avoided in favor of either independent aims (multiple different approaches to the same problem) or grant language and preliminary data 
suggesting that an aim's success is not in doubt, but rather that the aim seeks to 'refine' or 'develop' an already successful approach further.

Other possible reasons for lack of funding include an inappropriate study section assignment (for instance, a study section for basic science rather than neuroscience-specific research) or a lack of responsiveness to a specific Request for Applications (RFA) (when an application does not address the particular requirements of a funding mechanism). Both of the above can be prevented by communication with the PO. In fact, for most applications addressing important scientific or clinical problems, failure to obtain funding is often due to ineffective communication by the investigators.

\section{WHAT TO DO IF THE GRANT DID NOT GET FUNDED - THE RESUBMISSION AND RECYCLING PROCESS}

If an application is not funded, the investigator can choose between rebuttal, resubmission, and recycling.

\section{Rebuttal}

Setup a telephone meeting with the $\mathrm{PO}$ with a 1-2 page response to review. This may be sufficient to convert a queued application to a funded application, but is only suggested if the application is scored very well.

\section{Resubmission}

The advantage of resubmission is panel memory: it gives you a chance to improve your application based on the comments and recalibrate the scoring after revision. For the response to reviewers, list all the criticisms individually and come up with a solution to each of them. It is often helpful to group common themes in the response. After drafting the response (in the form of a single-page introduction), revise the rest of the application accordingly. We recommend resubmission to the same study section if you can address the criticisms appropriately and feel that the study section possessed the necessary expertise and background to correctly judge the application. Further preliminary data or figures to address specific criticism may strengthen a resubmission. Applications are only permitted a single resubmission; however, if the application is not funded after resubmission, it can be submitted again as a 'new' submission to the same study section.

\section{Recycling}

In consultation with your PO, the application can be resubmitted to either a different funding mechanism or a different study section. Alternatively, the grant can be resubmitted to another sponsor (such as a foundation or other federal agency) which may require more extensive revision of the application.

\section{CONCLUSIONS}

Successful grant submissions require detailed preparation in addition to proper scientific writing. Collaboration and persistence are critical to ultimate success. We hope this brief review will serve as a helpful guide for early-career neurointerventionalists who want to write their first grant application and begin a career of funded research.

Twitter Robert M Starke @Starke_neurosurgery and Michael R Levitt @ DrMichaelLevitt

Contributors PK: manuscript writing, manuscript editing, information research, project conceptualization; MM, WJM, RMS, KNS, FCA, MRL: manuscript editing, information research.

Funding The authors have not declared a specific grant for this research from any funding agency in the public, commercial or not-for-profit sectors.

Competing interests None declared.

Patient consent for publication Not required.

Provenance and peer review Commissioned; internally peer reviewed.

\section{ORCID iDs}

Peter Kan http://orcid.org/0000-0001-6649-4128

Maxim Mokin http://orcid.org/0000-0003-4270-8667

Michael R Levitt http://orcid.org/0000-0003-3612-3347

\section{REFERENCES}

1 NIH Grants and Funding website. NIH research project grant program (R01), 2019. Available: https://grants.nih.gov/grants/funding/r01.htm [Accessed 30 Sep 2020].

2 NIH National Institute of Allergy and Infectious Diseases website. Writing a winning application - draft and evaluate specific aims, 2020. Available: www.niaid.nih.gov/ grants-contracts/winning-application-specific-aims [Accessed 26 Sep 2020].

3 NIH National Institute of Allergy and Infectious Diseases website. Write your research plan, 2017. Available: www.niaid.nih.gov/grants-contracts/write-research-plan [Accessed 29 Sep 2020].

$4 \mathrm{NIH}$ Grants and Funding website. Definitions of criteria and considerations for research project grant (RPG/R01/R03/R15/R21/R34) critiques, 2016. Available: grants. nih.gov/grants/peer/critiques/rpg.htm [Accessed 26 Sep 2020].

5 Kallet RH. How to write the methods section of a research paper. Respir Care 2004;49:1229-32.

6 NIH Office of Extramural Research website. Project summary/abstract and project narrative: what's the difference and what to include, 2019. Available: nexus.od.nih gov/all/2019/06/28/project-summary-abstract-and-project-narrative-whats-thedifference-and-w [Accessed 28 Sep 2020].

$7 \mathrm{NIH}$ Grants and Funding website. Biosketch format pages, instructions and samples. Available: grants.nih.gov/grants/forms/biosketch.htm [Accessed 26 Sep 2020].

$8 \mathrm{NIH}$ National Institute of Allergy and Infectious Diseases website. For grant applicants - a focus on facilities and equipment, 2020. Available: www.niaid.nih.gov/ grants-contracts/facilities-and-equipment [Accessed 28 Sep 2020].

9 NIH National Institute of Allergy and Infectious Diseases website. Letters of support, 2020. Available: www.niaid.nih.gov/grants-contracts/letters-of-support [Accessed 28 Sep 2020].

10 NIH Office of Laboratory Animal Welfare website. Vertebrate animal section, 2020. Available: olaw.nih.gov/guidance/vertebrate-animal-section.htm [Accessed $30 \mathrm{Sep}$ 2020].

11 NIH Grants and Funding website. Human subjects research - home page, 2019. Available: grants.nih.gov/policy/humansubjects.htm [Accessed 29 Sep 2020].

12 NIH Grants and Funding website. Research sharing plan, 2015. Available: grants.nih. gov/grants/peer/guidelines_general/Resource_sharing_plans.pdf [Accessed $29 \mathrm{Sep}$ 2020].

13 NIH Grants and Funding website. Develop your budget, 2019. Available: grants.nih. gov/grants/how-to-apply-application-guide/format-and-write/develop-your-budget. htm [Accessed 30 Sep 2020].

14 NIH Grants and Funding website. Salary cap summary (FY 1990-Present), 2019 Available: grants.nih.gov/grants/policy/salcap_summary.htm [Accessed 30 Sep 2020].

15 NIH National Institute of Allergy and Infectious Diseases website. Review criteria SOP, 2020. Available: www.niaid.nih.gov/research/review-criteria [Accessed 29 Sep 2020].

16 NIH Center for Scientific Review website. Review process.. Available: public.csr.nih. gov/FAQs/ReviewersFAQs/ReviewProcess [Accessed September 29, 2020]. 